\title{
Heterozygous RFX6 protein truncating variants are associated with MODY with reduced penetrance
}

\author{
Kashyap A. Patel ${ }^{1}$, Jarno Kettunen (10 2,3,4, Markku Laakso5,6, Alena Stančáková (10 6, Thomas W. Laver ${ }^{1}$, \\ Kevin Colclough7, Matthew B. Johnson (10 1, Marc Abramowicz ${ }^{8}$, Leif Groop ${ }^{9,10}$, Päivi J. Miettinen (1) 11,12, \\ Maggie H. Shepherd ${ }^{1}$, Sarah E. Flanagan', Sian Ellard', Nobuya Inagaki ${ }^{13}$, Andrew T. Hattersley', \\ Tiinamaija Tuomi (1) 2,3,10, Miriam Cnop ${ }^{14,15}$ \& Michael N. Weedon ${ }^{1}$
}

Finding new causes of monogenic diabetes helps understand glycaemic regulation in humans. To find novel genetic causes of maturity-onset diabetes of the young (MODY), we sequenced MODY cases with unknown aetiology and compared variant frequencies to large public databases. From 36 European patients, we identify two probands with novel RFX6 heterozygous nonsense variants. RFX6 protein truncating variants are enriched in the MODY discovery cohort compared to the European control population within ExAC (odds ratio $=131$, $\left.P=1 \times 10^{-4}\right)$. We find similar results in non-Finnish European $(n=348$, odds ratio $=43$, $\left.P=5 \times 10^{-5}\right)$ and Finnish $\left(n=80\right.$, odds ratio $\left.=22, P=1 \times 10^{-6}\right)$ replication cohorts. RFX6 heterozygotes have reduced penetrance of diabetes compared to common HNF1A and HNF4A-MODY mutations (27, 70 and $55 \%$ at 25 years of age, respectively). The hyperglycaemia results from beta-cell dysfunction and is associated with lower fasting and stimulated gastric inhibitory polypeptide (GIP) levels. Our study demonstrates that heterozygous RFX6 protein truncating variants are associated with MODY with reduced penetrance.

\footnotetext{
${ }^{1}$ Institute of Biomedical and Clinical Science, University of Exeter Medical School, Exeter EX2 5DW, UK. ${ }^{2}$ Department of Endocrinology, Abdominal Centre, Helsinki University Hospital, Helsinki 00029, Finland. ${ }^{3}$ Folkhalsan Research Center, University of Helsinki, Helsinki 00014, Finland. ${ }^{4}$ Research Program of Diabetes and Obesity, Research Programs Unit, University of Helsinki, Helsinki 00014, Finland. ${ }^{5}$ Department of Medicine, Kuopio University Hospital, Kuopio 70029, Finland. ${ }^{6}$ Institute of Clinical Medicine, Internal Medicine, University of Eastern Finland, Kuopio 70029, Finland. ${ }^{7}$ Department of Molecular Genetics, Royal Devon and Exeter National Health Service Foundation Trust, Exeter EX2 5DW, UK. ${ }^{8}$ IRIBHM, Genetics Department, Erasmus Hospital, Université Libre de Bruxelles, Brussels 1070, Belgium. ${ }^{9}$ Lund University Diabetes Centre, Department of Clinical Sciences Malmö, Lund University, Skåne University Hospital, Malmö, SE 20502, Sweden. ${ }^{10}$ Institute for Molecular Medicine Finland (FIMM), University of Helsinki, Helsinki 00100, Finland. ${ }^{11}$ Children's Hospital, Pediatric Research Center, University of Helsinki and Helsinki University Hospital, Helsinki 00029, Finland. ${ }^{12}$ Molecular Neurology and Biomedicum Stem Cell Centre, Research Programs Unit, Faculty of Medicine, University of Helsinki, Helsinki 00014, Finland. ${ }^{13}$ Department of Diabetes, Endocrinology and Nutrition, Graduate School of Medicine, Kyoto University, Kyoto 606-8507, Japan. ${ }^{14}$ ULB Center for Diabetes Research, Faculty of Medicine, Université Libre de Bruxelles, Brussels 1070, Belgium. ${ }^{15}$ Division of Endocrinology, Erasmus Hospital, Université Libre de Bruxelles, Brussels 1070, Belgium. Kashyap A. Patel and Jarno Kettunen contributed equally to this work. Andrew T. Hattersley, Tiinamaija Tuomi, Miriam Cnop and Michael N. Weedon jointly supervised this work. Correspondence and requests for materials should be addressed to M.C. (email: mcnop@ulb.ac.be) or to M.N.W. (email: M.N.Weedon@exeter.ac.uk)
} 
F inding the genetic cause of rare familial diabetes (monogenic diabetes) provides new biological insights into human pancreas development and function, as well as potentially novel therapeutic targets with important treatment implications ${ }^{1}$. Maturity-onset diabetes of the young (MODY) is monogenic diabetes resulting from beta-cell dysfunction which usually present before the age of 25 years in non-obese patients who are non-insulin-dependent and have an autosomal dominant inheritance of diabetes ${ }^{2}$. Mutations in HNF1A, HNF4A and GCK are the commonest causes of MODY responsible for $\sim 60 \%$ of MODY aetiology ${ }^{1}$.

There has been limited recent success in finding new MODY genes. WFS1 heterozygous variants and loss-of-function variants in the APPL1 gene were shown to be a rare cause of MODY ${ }^{3,4}$. The reason for this limited success is the difficulty of distinguishing monogenic diabetes patients from those with type 1 diabetes $^{5,6}$, or from the increasing number of patients with earlyonset type 2 diabetes due to rising rates of obesity. Another important reason is the lack of large pedigrees with an autosomal dominant pattern of inheritance of diabetes which would allow classical linkage analysis to be performed and which was used to discover the most common forms of MODY such as GCK, HNF1A and HNF4A $A^{7-10}$.

Rare-variant association testing is an important step to confirm the pathogenicity of novel variants in monogenic disease ${ }^{11}$. Rarevariant association testing particularly for comparing the frequency of novel protein-truncating variants (PTVs) in monogenic cases with unknown aetiology to the frequency in large control cohorts is now possible because of the availability of resources such as ExAC - a database of protein coding variants in large control populations ${ }^{12}$. This allows burden testing of the frequency of novel or rare coding variants in diseases of interest and a comparison to rates in controls to identify new genetic causes of monogenic disease.

In this study, we have undertaken next-generation sequencing of MODY cases with unknown aetiology and compared the frequency of PTVs to large publicly available control cohorts to identify new MODY genes. Our study shows that heterozygous RFX6 PTVs are associated with MODY.

\section{Results}

Heterozygous RFX6 PTVs in MODY with unknown aetiology. To identify patients with novel heterozygous PTVs, we first assessed 38 European (non-Finnish) probands with a strong MODY-like phenotype who did not have mutations in the common MODY genes (GCK, HNF1A, HNF4A) by Sanger sequencing (Supplementary Table 1). To exclude the other known/less common causes of monogenic diabetes, these patients underwent comprehensive targeted-next generation sequencing (NGS) for all 29 known monogenic diabetes genes, including genes for neonatal diabetes, MODY and mitochondrial diabetes, lipodystrophy or other forms of syndromic diabetes ${ }^{13}$ (Supplementary Table 2). We identified two probands with mutations in the known MODY gene $H N F 1 B^{13}, 14$. The analysis of heterozygous PTVs in the 29 genes on the targeted panel identified two unrelated probands with a novel heterozygous nonsense variant in Regulatory Factor X 6 (RFX6) (Family 1 - p.Leu292Ter, Family 2 - p.Lys351Ter) (Table 1, Fig. 1 and Supplementary Table 3$)$. We did not identify any rare $(<1 \%)$ missense RFX6 variants in this cohort. RFX6 was part of the targeted sequencing panel because recessive RFX6 variants (missense and/or proteintruncating) are a known cause of syndromic neonatal diabetes ${ }^{15}$, but heterozygotes were not previously known to have any phenotype.

RFX6 PTVs are enriched in a MODY discovery cohort. We next compared the frequency of RFX6 PTVs in our discovery cohort to a large control population with whole-exome data from $\mathrm{ExAC}^{12}$. Neither of the RFX6 variants from the discovery cohort were present in the 60,706 individuals in ExAC. There were 15 individuals with RFX6 PTVs in the 33,346 ExAC non-Finnish European control population (Supplementary Table 3). The frequency of the RFX6 PTVs in the MODY discovery cohort was significantly higher (after accounting for the multiple testing of 29 genes) than the ExAC non-Finnish European control population (5.5 vs. $0.045 \%$, odds ratio (OR) 131 , 95\% confidence interval (CI) $14-595, P=1 \times 10^{-4}$ ) (Table 1).

RFX6 PTVs are enriched in a MODY replication cohort. To replicate the findings of our discovery cohort, we then examined 348 non-Finnish European probands who were routinely referred for MODY genetic testing to the Molecular Genetics Laboratory, Exeter, UK and in whom the common causes of MODY were excluded using targeted-NGS assay (Supplementary Table 1). The analysis of heterozygous PTVs identified four unrelated probands with two novel RFX6 nonsense variants (p.Gln25Ter, p.Arg377Ter) (Supplementary Fig. 1 and Supplementary Table 3). Similarly to the discovery cohort, the MODY replication cohort was enriched for RFX6 PTVs compared to the ExAC non-Finnish European control population ( 1.15 vs. $0.045 \%, \mathrm{OR}=26,95 \% \mathrm{CI}$ $6-82, P=3 \times 10^{-5}$ ) (Supplementary Table 4). This association was maintained when compared to an independent non-Finnish European control population with whole-genome sequence data from gnomAD (http://gnomad.broadinstitute.org) (Table 1 and Supplementary Table 4). The frequency of RFX6 PTVs in the gnom AD genome data set $(0.027 \%)$ is not statistically different to that in ExAC $(0.045 \%, P=0.76)$.

Higher frequency of RFX6 PTVs in Finnish population. Finnish individuals had $\sim 10$-fold higher frequency of RFX6 PTVs compared to non-Finnish Europeans. The ExAC database showed a relative abundance of RFX6 PTVs in Finnish Europeans $(15 / 3305,0.45 \%)$ compared to non-Finnish Europeans $(15 / 33,346,0.045 \%)$ (Supplementary Table 3). All of the Finnish individuals in ExAC with RFX6 PTVs had the same variant, p.His293Leufs. To further validate this finding in a larger Finnish control population, we analysed RFX6 PTVs in 7040 control individuals from the METSIM study in

Table 1 Frequency of heterozygous RFX6 protein-truncating variants in all study cohorts and control populations

\begin{tabular}{lllll} 
& MODY cohorts, frequency of $\boldsymbol{R F X 6}$ PTV & Control population, frequency of RFX6 PTV & Odds ratio (95\% CI) & P-value \\
\hline Non-Finnish European & Discovery cohort $(n=2 / 36), 5.5 \%$ & ExAC - exomes $(n=15 / 33,346), 0.045 \%$ & $131(14-595)$ & $1 \times 10^{-4}$ \\
& Replication cohort $(n=4 / 348), 1.15 \%$ & gnomAD - genomes $(n=2 / 7508), 0.027 \%$ & $43(6-483)$ & $5 \times 10^{-5}$ \\
Finnish European & Replication cohort $(n=6 / 80), 7.5 \%$ & METSIM-exomes $(n=26 / 7040), 0.37 \%$ & $22(7-56)$ \\
Meta-analysis Heterogeneity $\left(\chi^{2}=2.73(\right.$ d.f. $\left.=2), P=0.256\right)$ & & $34(15-80)$ \\
\hline Cl, confidence interval; MODY, maturity-onset diabetes of the young; PTV, protein-truncating variant & $1 \times 10^{-6}$ \\
\hline
\end{tabular}




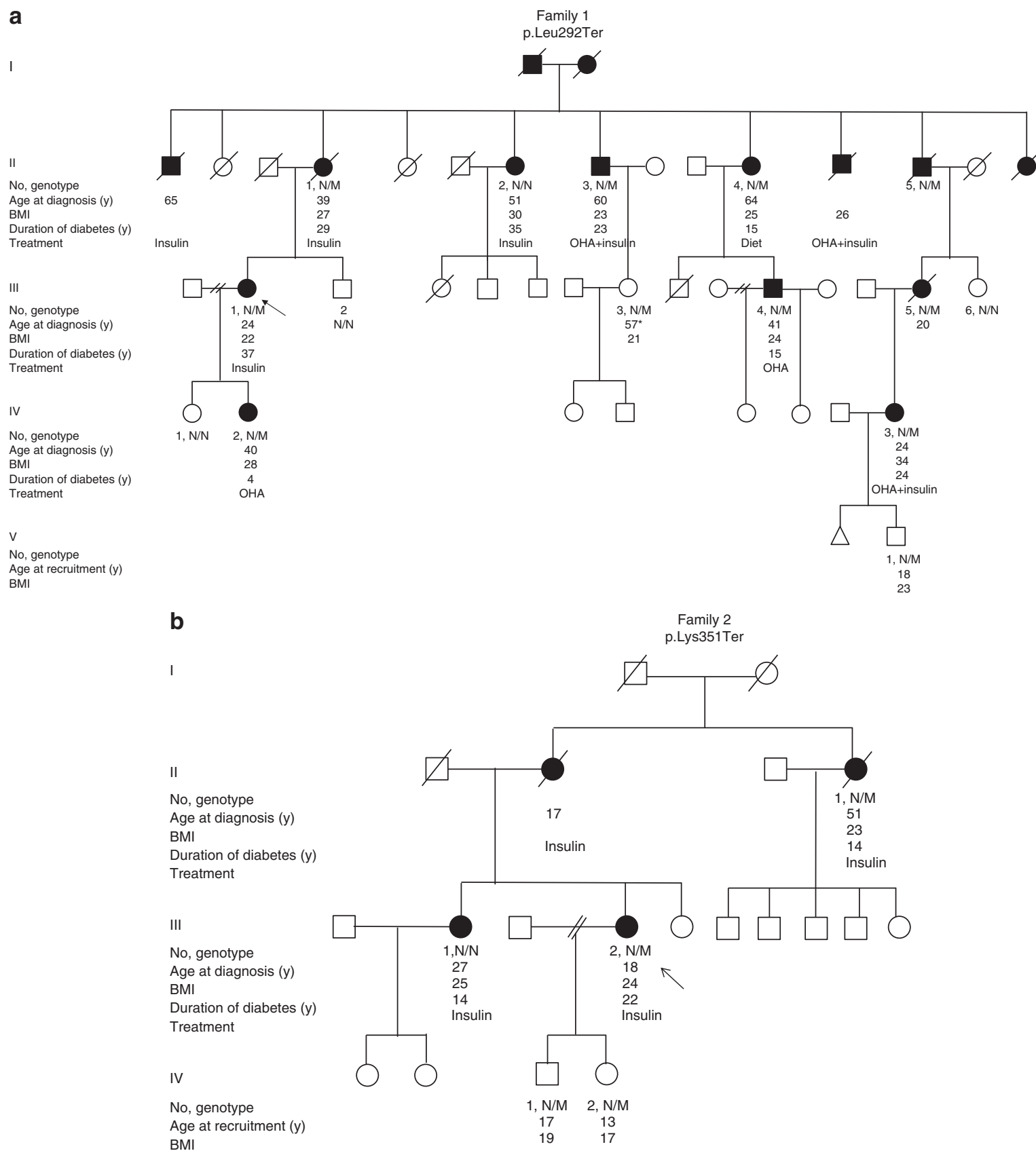

Fig. 1 Extended pedigree of non-Finnish European patients identified in the discovery cohort. a Pedigree of family 1 that were identified with heterozygous RFX6 variant (NM_173560.3:c.875-T> G,p.Leu292Ter) from the discovery cohort. b Pedigree of family 2 from the discovery cohort with heterozygous RFX6 variant (NM_173560.3:c.1051-A > T, -p.Lys351Ter). Genotype is shown underneath each symbol; $\mathrm{M}$ and $\mathrm{N}$ denote mutant and wild-type alleles, respectively. Directly below the genotype is the age of diabetes onset in years, duration in years, BMI and treatment at study entry. Squares represent male family members, and circles represent female members. Black-filled symbols denote patients with diabetes. An arrow denotes the proband in the family. $\mathrm{OHA}$, oral hypoglycaemic agents. *age at recruitment. One of the daughters of patient III.1 in family 2 had a history of gestational diabetes

Eastern Finland ${ }^{16}$. There were 26 individuals with RFX6 PTVs in this cohort and all had the p.His293Leufs variant. The frequency of p.His293Leufs was not significantly different from the ExAC Finnish population frequency ( 0.37 vs. $0.45 \%$, $P=0.63$ ) (Supplementary Table 3 ). The METSIM study has contributed to the ExAC Finnish cohort, so to prevent duplication we used the data from the larger METSIM study for further analysis ${ }^{12}$.

Enrichment of RFX6 p.His293Leufs in Finnish MODY patients. To assess whether the p.His293Leufs variant is associated with MODY in Finnish patients, we genotyped the RFX6 p.His293Leufs variant in 80 Finnish probands who were routinely referred for MODY genetic testing to Genome Center of Eastern Finland, University of Eastern Finland and did not have mutations in the most common MODY genes (GCK, HNF1A, HNF4A and HNF1B) (Supplementary Table 1). We identified six probands with the p.His293Leufs variant. The frequency of this variant was significantly higher in the Finnish MODY cohort compared to the METSIM controls (7.5 vs. $0.37 \%, \mathrm{OR}=22,95 \%$ CI 7-56, $P=1 \times 10^{-6}$ ) (Table 1). The meta-analysis of the three independent case-control analyses confirmed the strong 
association of RFX6 PTVs with MODY in the study cohorts (OR $=34,95 \%$ CI $15-80, P=1 \times 10^{-16}$ ) (Table 1$)$.

Enrichment of RFX6 PTVs is not due to technical artefacts. To ensure that the association we observed is not due to differences in sequencing technologies or analysis pipelines between cases and controls, we performed a series of sensitivity analyses. This included comparisons to additional whole exome, whole genome and in-house control cohorts and an analysis that removed exon 1 which was the least well covered exon in ExAC. These sensitivity analyses (Supplementary Table 4) show that results are consistent for all these analyses.

RFX6 PTVs co-segregate with diabetes. To further assess the causality of RFX6 PTVs, we conducted a co-segregation analysis in families with genetic data available on more than three affected individuals. We had only one family (family 1) with $>3$ affected individuals with genetic data (Fig. 1) ${ }^{17}$. The analysis showed that the RFX6 variant p.Leu292Ter co-segregated in 9 out of 10 individuals with diabetes (LOD score $=0.65, P=0.04$ ). One individual without the RFX6 variant had diabetes which is likely to be a phenocopy of type 2 diabetes considering the large pedigree, age of diagnosis and obesity (51 years, body mass index (BMI) $30 \mathrm{~kg} / \mathrm{m}^{2}$ ). There were two family members with an RFX6 variant but with normal HbA1c level at the time of study (18 and 57 years) suggesting that RFX6 PTVs may have reduced penetrance.

Reduced penetrance of diabetes with RFX6 PTVs. To assess the penetrance of RFX6 PTVs for diabetes compared to common causes of MODY, we combined data for all six non-Finnish European proband families. There were 18 RFX6 heterozygotes of whom five had not developed diabetes at study entry. $27 \%$ (95\% CI 11-58) developed diabetes by the age of 25 years and $78 \%$ (95\% CI 55-95) by 51 years (Fig. 2). Two out of six probands did not have affected parents at study entry (Supplementary Fig. 1). The penetrance of diabetes for RFX6 heterozygotes was substantially lower compared to pathogenic variants of HNF1A (70\%, $95 \%$ CI $67-72$ by the age of 25 years and $97 \%$, 95\% CI $96-98$ by 50 years) and moderately lower than pathogenic variants of HNF4A (55\%, 95\% CI 50-60 by the age of 25 years and 91\%, 95\% CI 88-94 by 50 years) (Fig. 2). Similar to non-Finnish European proband families, the Finnish RFX6 p.His293Leufs variant also showed reduced penetrance in Finnish families (Supplementary Fig. 2). In two previously reported families of neonatal diabetes

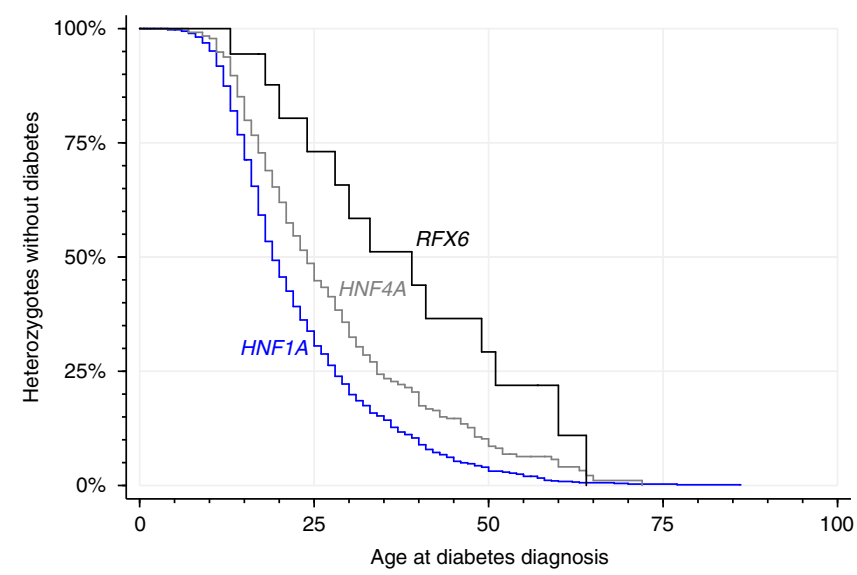

Fig. 2 Penetrance of diabetes in people with MODY. Heterozygous RFX6 PTV $(n=18)$, pathogenic HNF1A variant $(n=1265)$ or HNF4A variant $(n=$ 427) children with homozygous p.Arg181Gln RFX6 $6^{15,18}$ or RFX6 p. His293Leufs ${ }^{19}$, the genetic information available on RFX6 heterozygous family members was also suggestive of reduced penetrance of diabetes (Supplementary Figs 2 and 3).

RFX6 PTVs are not enriched in type 2 diabetes. The reduced penetrance and later age of onset of diabetes with RFX6 PTVs raised the possibility that these variants may be associated with type 2 diabetes. To assess this, we used freely available data from the Type 2 Diabetes Knowledge Portal which contains wholeexome data on type 2 diabetes patients ${ }^{20}$. Burden testing of RFX6 PTVs for exome sequencing data from 8373 type 2 diabetes cases and 8466 controls showed no significant association with type 2 diabetes $(0.14 \text { vs. } 0.083 \%, \mathrm{OR}=1.79,95 \% \text { CI } 0.7-4.57, P=0.22)^{20}$ (Supplementary Table 3).

Phenotype of RFX6 heterozygotes with diabetes. We assessed the diabetes phenotype in 27 RFX6 heterozygote individuals with diabetes. The clinical features are shown in Table 2. The median age at diagnosis of diabetes was 32 years (IQR 24-46, range 13-64 years) and median BMI of $25.1 \mathrm{~kg} / \mathrm{m}^{2}$ (IQR 23-28). After a median 10 years (IQR 5-22) of diabetes $69 \%$ of patients were treated with insulin but there was significant endogenous insulin present in 24/25 patients at recruitment. There was no history of sulphonylurea sensitivity and they did not have islet autoantibodies (GADA/IA2-Ab). All patients had isolated diabetes and there were no reports of the other features of homozygous RFX6 mutations, such as duodenal or gall bladder atresia.

RFX6 haploinsufficiency is associated with reduced GIP. RFX6 is a transcription factor and has been shown to increase expression and secretion of gastric inhibitory polypeptide (GIP) in mouse enteroendocrine $\mathrm{K}$-cells ${ }^{21}$. We therefore measured the incretin hormone GIP in 17 RFX6 heterozygotes (eight with diabetes) and compared to 26 controls (two with diabetes). The fasting GIP was markedly lower in RFX6 heterozygotes compared to controls (16 (10-24) vs. $\left.49(28-65) \mathrm{pg} \mathrm{ml}^{-1}, P=1.2 \times 10^{-5}\right)$. Fasting glucagon-like peptide-1 (GLP-1) levels were not different in both groups $\left(23\right.$ (12.5-32) vs. $\left.24(14-32) \mathrm{pg} \mathrm{ml}^{-1}, P=0.98\right)$. To

Table 2 Clinical characteristics of patients with RFX6-MODY$$
\text { Bur }
$$

$\begin{array}{ll}\text { Characteristic } & (n=27) \\ \text { Age at diagnosis (years), median (IQR) } & 32(24-46) \\ \text { Duration of diabetes, median (IQR) } & 10(5-22) \\ \text { Female, } n(\%) & 22(81 \%) \\ \text { BMI }\left(\mathrm{kg} / \mathrm{m}^{2}\right) \text {, median (IQR) } & 25.1(23-28) n=22 \\ \text { Initial treatment, } n(\%) & \\ \text { Diet } & 4(15 \%) \\ \text { Oral hypoglycaemic agents } & 14(54 \%) \\ \text { Insulin } & 5(19 \%) \\ \text { Insulin + oral hypoglycaemic agents } & 3(12 \%) \\ \text { Current treatment, } n \text { (\%) } & \\ \text { Diet } & 2(8 \%) \\ \text { Oral hypoglycaemic agents } & 6(23 \%) \\ \text { Insulin } & 12(46 \%) \\ \text { Insulin + oral hypoglycaemic agents } & 6(23 \%) \\ \text { HbA1c at recruitment, mmol/mol, median (IQR) } & 51(45-70) n=22 \\ \text { Significant endogenous insulin at recruitment }{ }^{\mathrm{a}}, & 24(96 \%) n=25 \\ n(\%) & \\ & \end{array}$

$\mathrm{BMI}$, body mass index; IQR, interquartile range; MODY, maturity-onset diabetes of the young aNon-insulin treated or insulin treated with urine/blood random C-peptide $>200 \mathrm{pmol} / \mathrm{I}$ at recruitment 
a

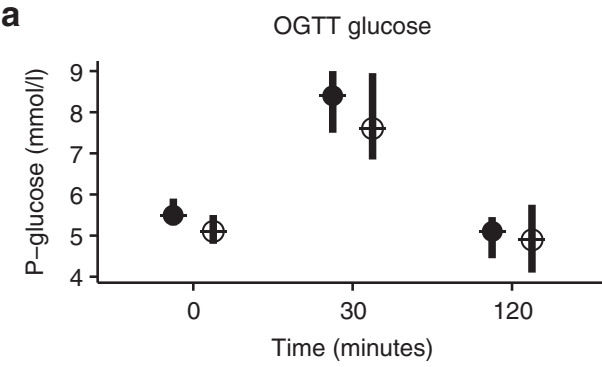

C

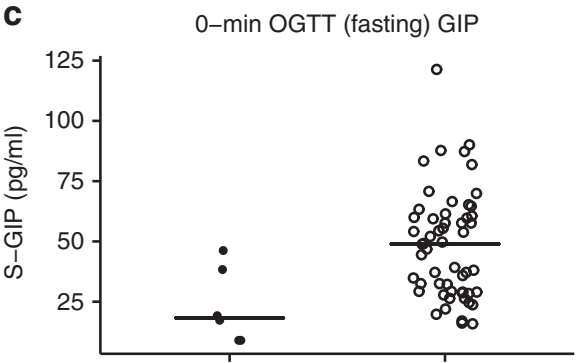

e

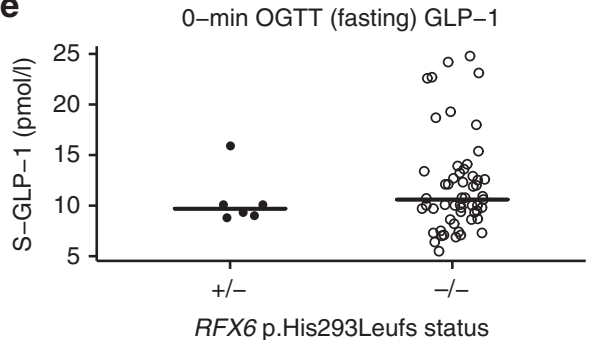

b

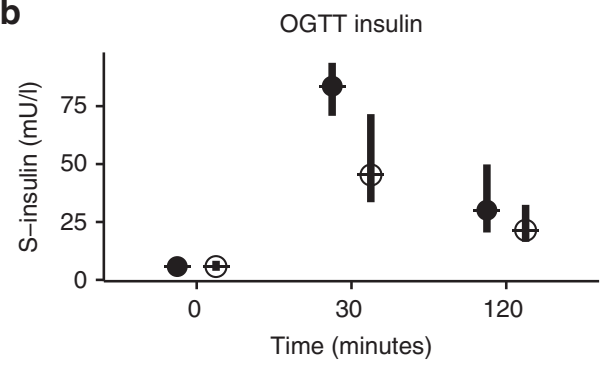

d

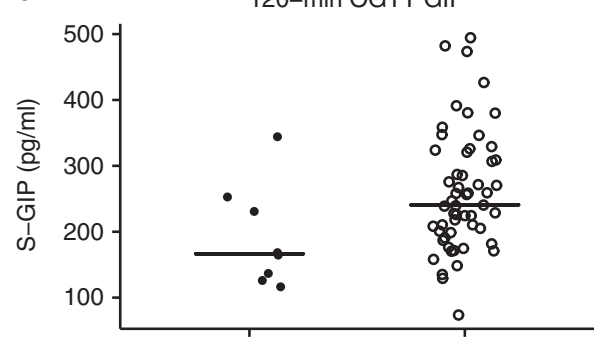

f

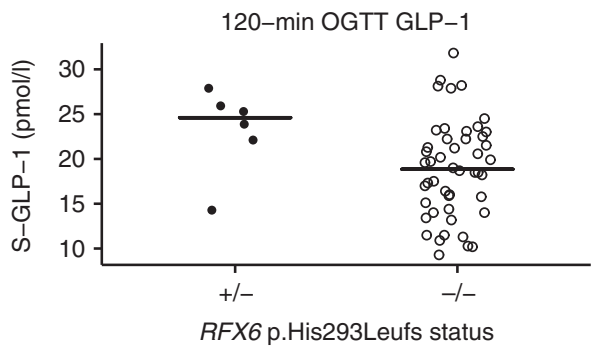

Fig. 3 Phenotypic characteristics of the Finnish RFX6 p. His293Leufs heterozygotes without diabetes. Figure shows the phenotypic characteristics at fasting and during an oral glucose tolerance test (OGTT) of the Finnish RFX6 p. His293Leufs heterozygotes without diabetes (filled symbols; $n=11$ ) compared with population controls from the PPP-Botnia Study (open symbols; $N=55$ ) matched for age, sex and BMI. a Median and interquartile range of plasma glucose concentrations during an OGTT ( $N=11$ vs. 55). b Median and interquartile range of serum insulin concentrations during an OGTT ( $N=11$ vs. 55). c Individual fasting GIP concentration for RFX6 heterozygotes and controls ( $N=6$ vs. 55). d Individual 120-minute GIP concentration during an OGTT ( $N=8$ vs. 55 ). e Individual fasting GLP-1 concentration ( $N=6$ vs. 55 ). $\mathbf{f}$ Individual 120 min GLP-1 concentration during an OGTT ( $N=6$ vs. 55 ). The $P$-values (Mann-Whitney $U$ test) under 0.05 for the difference in a Glucose $0 \mathrm{~min}, P=0.02$; $\mathbf{b}$ Insulin $30 \mathrm{~min}, P=0.015 ; \mathbf{c}$ fasting GIP, $P=8.6 \times 10^{-3} \mathbf{d} 120$ minute GIP, $P=0.029$; e 120-minute GLP-1, $P=0.047$; $+/-$, heterozygous for RFX6 variant; $-/-$, controls without RFX6 variant. Horizontal black line in panels c-f represents median for each group

remove potential confounding factors, we compared the OGTT data for the 11 Finnish RFX6 p.His293Leufs heterozygotes without diabetes to five matched (age, sex and BMI) controls for each heterozygote from the PPP-Botnia Study (Fig. 3 and Supplementary Table 6). This confirmed that both fasting and $120 \mathrm{~min}$ stimulated GIP was reduced (18.3 vs. $48.9 \mathrm{pg} \mathrm{ml}^{-1}$, $P=8 \times 10^{-3}, 167$ vs. $241 \mathrm{pg} \mathrm{ml}^{-1}, P=0.029$, respectively). In addition, the non-diabetic heterozygotes had higher fasting glucose (5.5 vs. $5.1 \mathrm{mmol} \mathrm{l}^{-1}, P=0.02$ ) with a similar fasting insulin level suggesting a beta-cell defect (Supplementary Table 6).

\section{Discussion}

Heterozygous RFX6 PTVs are associated with MODY. We identified RFX6 PTVs predicted to be pathogenic ${ }^{11,19}$ in unrelated MODY patients in whom the known causes of monogenic diabetes had been excluded. These variants were enriched in Finnish and non-Finnish European MODY probands but were rare in the control cohorts and in patients with type 2 diabetes. We observed co-segregation within pedigrees albeit with reduced penetrance. Finally, these variants are likely to have a functional effect due to nonsense-mediated decay causing haploinsufficiency ${ }^{12,22}$. This is further supported by the studies that showed that the homozygous RFX6 PTVs cause neonatal diabetes ${ }^{15}, 23,24$. Among unknown
MODY cases, RFX6 PTVs were responsible for 7.5\% Finnish cases compared to only $\sim 1 \%$ of non-Finnish European cases.

Large-scale control cohorts such as ExAC in combination with next-generation sequencing of well-characterised cases is a useful strategy for identifying new causes of monogenic disease. The large ExAC database provides sufficient power for reliable burden testing of rare variants in monogenic disease ${ }^{12}$ without the need for large pedigrees or linkage analysis. RFX6 PTVs were highly enriched in both discovery and replication cohorts compared to control cohorts, supporting their pathogenicity. The ExAC database has been very useful in identifying benign variants because of an unusually high frequency in the population compared to frequency of the disease in question $11,12,25$. However, caution is required for reduced penetrance variants as their frequency can be higher than estimated disease frequency in the general population. RFX6 PTVs are an example where the reduced penetrance explains the higher frequency in control cohorts compared to the estimated frequency of MODY $(0.01 \%)$ in the general population $^{26}$. In addition, our study highlights the importance of population specific control and disease cohorts. The frequency of RFX6 PTVs is $\sim 10$-fold higher in the Finnish population compared to non-Finnish populations due to the well-documented bottleneck in population genetics ${ }^{27,} 28$. This observation is not 
restricted to RFX6 alone. It has been shown that the Finnish population has an overall higher burden of genome-wide PTVs $(0.5-5 \%)$ in many genes compared to non-Finnish Europeans, and some of these have been associated with disease $\mathrm{e}^{27-29}$.

RFX6 PTVs are associated with reduced penetrance MODY. This reduced penetrance explains the lack of complete co-segregation in the RFX6 pedigrees. It also clarifies why diabetes was only reported in the parents or grandparents (obligate heterozygous for functional RFX6 variants) in seven out of the 12 published recessive RFX6 neonatal diabetes pedigrees $15,18,24,30-36$. The lack of enrichment of RFX6 PTVs in type 2 diabetes patients compared to controls further supports their association with reduced penetrance MODY rather than type 2 diabetes. Further studies are needed to understand the mechanism of reduced penetrance of diabetes in RFX6 heterozygotes. It could be due to a combination of factors, such as expression patterns of normal alleles, epigenetic modifications and rare or common genetic variant modifiers ${ }^{37}$.

There are differences as well as similarities between RFX6MODY and HNF1A/HNF4A-MODY. In contrast to HNF1A and HNF4A-MODY patients, RFX6-MODY patients do not show enhanced sensitivity to sulphonylureas ${ }^{38}$. All patients with HNF1A/HNF4A-MODY have significant endogenous insulin 3-5 years post diagnosis ${ }^{39}$. RFX6-MODY patients showed a similar pattern, except for one patient who did not have detectable endogenous insulin. Similar to HNF1A/HNF4A-MODY, 38, RFX6-MODY patients lack islet autoantibodies and have isolated diabetes. This suggests that persistent C-peptide, lack of islet autoantibodies and parental history of diabetes, which are currently used to distinguish common forms of MODY from type 1 diabetes, can also be used to identify RFX6-MODY. However, a similar strategy will not help to distinguish late onset RFX6MODY from type 2 diabetes.

Our study supports the role of RFX6 in the adult human pancreas. RFX6 is from a family of transcription factors that contains winged-helix DNA-binding domains ${ }^{15}$. RFX6 is expressed almost exclusively in pancreatic islets, small intestine and $\operatorname{colon}^{15}$. It acts downstream of NGN3, regulates islet cell differentiation and the development of the endocrine pancreas ${ }^{15}$. The homozygous RFX6 missense and PTVs cause syndromic neonatal diabetes (gall bladder aplasia, gut atresia and diabetes) ${ }^{15}$. RFX6 whole-body null mice show phenotypes consistent with human disease and die soon after birth, but the heterozygous whole-body RFX6 mouse has not been reported to develop diabetes ${ }^{15}$. This is not surprising considering the lack of phenotype in heterozygous null mice of $H N F 1 A$ and $H N F 1 B^{40}$. Interestingly, the defect in glucose-induced insulin secretion was present in models that are more akin to haploinsufficiency of RFX6 in adult humans ${ }^{33}, 41.80 \%$ depletion of RFX6 protein in the adult mouse pancreas in vivo as well as in human beta cells in vitro showed that this defect was due to reduced expression of $\mathrm{ABCC}$, GCK and $\mathrm{Ca}^{2+}$ channels in beta cells and disruption of $\mathrm{Ca}^{2+}$-mediated insulin secretion ${ }^{33,41}$. These data support the role of RFX6 in the physiology of adult beta cells. This along with evidence of impaired of beta-cell function (requirement of insulin to maintain euglycaemia, one patient with C-peptide $<200 \mathrm{pmoll}^{-1}$, lower C-peptide in heterozygotes with diabetes compared to without diabetes (Supplementary Fig. 4)), suggest that insulin deficiency is the cause of diabetes in these patients.

Incretins are gut hormones released in response to meals that potentiate glucose-stimulated insulin secretion. GIP is secreted from enteroendocrine $\mathrm{K}$-cells in the duodenum and upper jejunum and mediates the bulk of the incretin effect in healthy individuals ${ }^{42}$. The secretion of GIP and GLP-1 is preserved in type 2 diabetic patients ${ }^{43,44}$ and in patients with other forms of diabetes, including type 1 diabetes $^{45}$ and HNF1A-MODY ${ }^{46}$.
The present identification of GIP deficiency in RFX6 PTV heterozygotes is in keeping with the murine data showing that GIP expression and secretion is regulated by $\mathrm{Rfx}^{21}{ }^{21}$, and, importantly, identifies the first human form of diabetes associated with decreased GIP secretion.

In conclusion, heterozygous RFX6 PTVs are associated with reduced penetrance MODY and GIP deficiency.

\section{Methods}

Study populations. Discovery MODY cohort: The discovery cohort comprises 38 European probands with strong MODY-like phenotype who did not have mutations in the three most common MODY genes (GCK, HNF1A and HNF4A) (Supplementary Table 1 ). They were diagnosed $<25$ years of age, non-obese, had $\geq 3$ generation history of diabetes, non-insulin treated or insulin treated with C-peptide $>200$ pmol l$^{-1}$ (if available) and lacked islet autoantibodies.

Non-Finnish European replication MODY cohort: The replication cohort was derived from 469 non-Finnish European routine MODY diagnostic referrals to the Molecular Genetic Laboratory, Exeter, UK. A monogenic aetiology in a known monogenic diabetes gene was identified in 121 patients and the remaining 348 patients with unknown aetiology comprised the replication cohort (Supplementary Table 1).

Finnish-European replication MODY cohort: This cohort consisted of 80 patients who were routinely referred for MODY diagnostic testing to the Genome Center of Eastern Finland, University of eastern Finland in whom no mutation was found in the common MODY genes (GCK, HNF1A, HNF1B and HNF4A) when assessed by Sanger sequencing (Supplementary Table 1). These 80 patients comprise $78 \%$ of the total MODY X Finnish cohort.

Finnish-European control cohort: Individuals of this cohort were part of the METSIM study $(n=7040)$. They were all males aged $45-70$ years, randomly selected from the population register of the Kuopio town, Eastern Finland ${ }^{16}$

Cohort of people with pathogenic HNF1A and HNF4A variants: This cohort included probands and their family members referred to the Molecular Genetics Laboratory, Exeter, UK for MODY genetic testing and were identified to have a pathogenic HNF1A $(n=1265)$ or HNF4A $(n=427)$ variant.

Phenotypic characterisation of RFX6 heterozygotes: In total, we had 47 RFX6 heterozygotes of whom 27 had diabetes. 29/47 were part of the discovery and replication cohorts. 18/47 were identified separately or had been previously reported $^{18,23}$ (Supplementary Fig. 2 - Families 3-5, Supplementary Fig. 3). The clinical features of RFX6-MODY were based on 27 individuals with diabetes. 13/27 were part of the discovery and non-Finnish replication cohort (Fig. 1,

Supplementary Fig. 1 - Families 1-6). 9/27 individuals were from the Finnish replication cohort (5/9 individuals were from Supplementary Fig. 2 - Families 1 and 2, Pedigrees were not available for $4 / 9$ individuals). In addition to this, we included five diabetic individuals that were identified separately. This comprised a single Finnish individual (Supplementary Fig. 2 - Family 3) and four individuals from a previously reported family from Belgium (Supplementary Fig. 3 ) $^{18}$.

Incretin analysis: We completed physiological studies on 25/47 RFX6

heterozygotes in whom 10 had diabetes. $7 / 25$ had fasting blood sample analysis and $18 / 25$ had $75 \mathrm{~g}$ oral glucose tolerance test (OGTT). We used 27 (12 family and 15 unrelated) controls that included two individuals with diabetes for an initial comparison of fasting GIP/GLP-1. RFX6 heterozygotes had similar age (38 (34-58) vs. $40(30-61)$ years, $P=0.70$ ), sex (female 64 vs. $56 \% P=0.58$ ) and BMI (24 (23-29) vs. $\left.27(24-30) \mathrm{kg} / \mathrm{m}^{2}, P=0.27\right)$ distribution as the 27 controls. Fasting GIP/GLP-1 levels were available on 17/25 RFX6 heterozygotes and 26/27 controls. Out of 25 individuals who were assessed for GIP/GLP-1 analysis, 16 Finnish individuals (five with diabetes, 11 without diabetes) participated in the FINNMODY Study (www.botnia-study.org/finnmody, recruiting patients with MODY-like diabetes and their relatives in Finland). It is linked to the population based PPP-Botnia study and the participants had been subjected to standardised OGTT, sample collection and detailed biochemical analysis following the study protocol of the PPP-Botnia study. Therefore, to remove any potential confounding factors, we compared the phenotypic characteristics of these 11 RFX6 p. His293Leufs heterozygotes without diabetes to five controls for each heterozygote from the PPP-Botnia study matched for age, sex and BMI. None of the controls had the RFX6 p.His293Leufs variant.

PPP-Botnia Study: The Prevalence, Prediction and Prevention of diabetes (PPP)-Botnia Study is a population-based study in Western Finland aiming at obtaining accurate estimates of prevalence and risk factors for type 2 diabetes, impaired glucose tolerance, impaired fasting glucose and the metabolic syndrome in the adult population (Isomaa). Altogether 5208 individuals randomly recruited from the national Finnish Population Registry participated in the baseline study in 2004-2008 (representing 6-7\% of the population), and 3870 (77\%) individuals participated in the follow-up study in 2011-2014. The participants with fasting plasma glucose $<10 \mathrm{mmol}^{-1}$ participated in an $75 \mathrm{~g}$ OGTT with venous samples taken at $0,30,120 \mathrm{~min}$ for plasma glucose and serum insulin; at 0 and $120 \mathrm{~min}$ for serum C-peptide, GIP and GLP-1. The participants gave their written informed consent and the study protocol was approved by the Ethics Committee of Helsinki University Hospital, Finland. 
Plasma glucose was analysed using the Hemocue Glucose System (HemoCue AB, Ängelholm, Sweden). Serum insulin was measured by an AutoDelfia fluoroimmunometric assay (PerkinElmer, Waltham, Massachusetts, US) and serum C-peptide by Cobas e411 electrochemiluminometric immunoanalysis (Roche, Mannheim, Germany). Serum GIP was analysed using Millipore's Human GIP Total ELISA (Merck, Darmstadt, Germany; catalogue \# EZHGIP-54K), which has $100 \%$ cross-reactivity to both human GIP (1-42) and GIP (3-42). Serum total plasma GLP-1 concentrations, which detects both intact GLP-1 and GLP-1 (9-36 amide), were determined using Millipore's radioimmunoassay (Merck, Darmstadt, Germany; catalogue \#GLP1T-36HK). Serum total cholesterol, highdensity lipoprotein and triglyceride concentrations were measured first on a Cobas Mira analyser (Hoffman LaRoche, Basel, Switzerland) and low-density lipoprotein cholesterol concentrations were calculated using the Friedewald formula, and since January 2006 with an enzymatic method (Konelab 60i analyser; Thermo Electron Oy, Vantaa, Finland)

The RFX6 p.His293Leufs variant was genotyped in 5187 individuals by the Kompetitive Allele Specific PCR genotyping system (KASPTM) on Demand (KOD) assay according to the manufacturer's testing conditions including six positive control samples identified by direct sequencing (LGC Hoddesdon, Herts, UK). Two out of 5180 participants had RFX6 p.His293Leufs (the genotyping failed in 7), which was confirmed by direct sequencing.

DNA analysis. Targeted next-generation sequencing: The analysis of all known monogenic diabetes genes in European cohorts was conducted using targeted$\mathrm{NGS}^{13}$. The panel included 29 genes in which variants are known to cause monogenic neonatal diabetes, MODY, mitochondrial diabetes, lipodystrophy or other forms of syndromic diabetes ${ }^{13}$ (Supplementary Table 2). The RFX6 PTVs identified by targeted-NGS were confirmed using Sanger sequencing. The essential splice site, nonsense and frameshift variants excluding the last exon were considered PTVs in this study ${ }^{12,22}$. The targeted-NGS assay covered $100 \%$ bases of the RFX6 coding region with $>10 \times$ read depth for all the samples

Sanger sequencing: Genomic DNA was extracted from whole blood using standard procedures and the coding region and intron/exon boundaries of the RFX6 gene were amplified by PCR. Amplicons were sequenced using the Big Dye Terminator Cycler Sequencing Kit v3.1 (Applied Biosystems, Warrington, UK) according to manufacturer's instructions and reactions were analysed on an ABI 3730 Capillary sequencer (Applied Biosystems, Warrington, UK). Sequences were compared with the reference sequences (NM_173560.3) using Mutation Surveyor v3.24 software (So Genetics, State College, PA, USA).

The Finnish-European MODY cohort was analysed for p.His293Leufs variant using Sanger sequencing as described above. Family co-segregation analysis was performed in available family members using a Sanger sequencing assay for the specific RFX6 variant identified in that family. DNA analysis of the METSIM study has been described before ${ }^{23}$.

Statistical analysis. Fisher's exact test was used to compare the frequency of RFX6 PTVs. The threshold $P$-value for association was $1 \times 10^{-3}$ as there were 29 genes on the panel $(0.05 / 29)$. The penetrance of diabetes was assessed using survival time analysis method. The statistical analysis was conducted using Stata 14 (StataCorp, Texas, USA). The comparison of RFX6 heterozygotes to PPP-Botnia controls were conducted using R (3.3.2) with packages for the data manipulation (dplyr) and visualization (ggplot2). Continuous variables were compared with Mann-Whitney $U$-test and categorical variables with chi-squared test. Single point non-parametric linkage analyses were performed using MERLIN 1.1.2 ${ }^{47}$. The $Z$-score was converted into a LOD score by use of the Kong and Cox exponential model implemented in MERLIN ${ }^{47}, 48$.

Ethics. Informed consent was obtained from all subjects. The UK study is approved by the North Wales Research Ethics Committee. The FINNMODY/PPPBotnia study is approved by the Research Ethics committee for Medicine of the Helsinki University Hospital.

Data availability. The majority of data used in this study are publically available and can be accessed via the studies cited in the text. Considering issues of patient confidentiality and restrictions in IRB permissions, other original data are available through specific request.

Received: 19 January 2017 Accepted: 4 August 2017

Published online: 12 October 2017

\section{References}

1. McCarthy, M. I. \& Hattersley, A. T. Learning from molecular genetics: novel insights arising from the definition of genes for monogenic and type 2 diabetes. Diabetes 57, 2889-2898 (2008).
2. Murphy, R., Turnbull, D. M., Walker, M. \& Hattersley, A. T. Clinical features diagnosis and management of maternally inherited diabetes and deafness (MIDD) associated with the $3243 \mathrm{~A}>\mathrm{G}$ mitochondrial point mutation. Diabet. Med. 25, 383-399 (2008).

3. Bonnycastle, L. L. et al. Autosomal dominant diabetes arising from a Wolfram syndrome 1 mutation. Diabetes 62, 3943-3950 (2013).

4. Prudente, S. et al. Loss-of-function mutations in APPL1 in familial diabetes mellitus. Am. J. Hum. Genet. 97, 177-185 (2015).

5. Oram, R. A. et al. Most people with long-duration type 1 diabetes in a large population-based study are insulin microsecretors. Diabetes Care 38, 323-328 (2015).

6. McDonald, T. J. et al. Islet autoantibodies can discriminate maturity-onset diabetes of the young (MODY) from type 1 diabetes. Diabet. Med. 28, 1028-1033 (2011).

7. Froguel, P. et al. Close linkage of glucokinase locus on chromosome $7 \mathrm{p}$ to earlyonset non-insulin-dependent diabetes mellitus. Nature 356, 162-164 (1992).

8. Hattersley, A. T., Turner, R. C., Patel, P. \& O'Rahilly, S. Linkage of type 2 diabetes to the glucokinase gene. Lancet 339, 1307-1310 (1992).

9. Yamagata, K. et al. Mutations in the hepatocyte nuclear factor-1alpha gene in maturity-onset diabetes of the young (MODY3). Nature 384, 455-458 (1996).

10. Yamagata, K. et al. Mutations in the hepatocyte nuclear factor-4alpha gene in maturity-onset diabetes of the young (MODY1). Nature 384, 458-460 (1996).

11. MacArthur, D. G. et al. Guidelines for investigating causality of sequence variants in human disease. Nature 508, 469-476 (2014)

12. Lek, M. et al. Analysis of protein-coding genetic variation in 60,706 humans. Nature 536, 285-291 (2016).

13. Ellard, S. et al. Improved genetic testing for monogenic diabetes using targeted next-generation sequencing. Diabetologia 56, 1958-1963 (2013).

14. Shepherd, M. et al. Systematic population screening, using biomarkers and genetic testing, identifies $2.5 \%$ of the U.K. pediatric diabetes population with monogenic diabetes. Diabetes Care 39, 1879-1888 (2016).

15. Smith, S. B. et al. Rfx6 directs islet formation and insulin production in mice and humans. Nature 463, 775-780 (2010).

16. Stančáková, A. et al. Changes in insulin sensitivity and insulin release in relation to glycemia and glucose tolerance in 6,414 Finnish men. Diabetes 58 1212-1221 (2009).

17. Frayling, T. M. et al. A genome-wide scan in families with maturity-onset diabetes of the young. Diabetes 52, 872-881 (2003).

18. Martinovici, D. et al. Neonatal hemochromatosis and Martinez-Frias syndrome of intestinal atresia and diabetes mellitus in a consanguineous newborn. Eur. J. Med. Genet. 53, 25-28 (2010).

19. Richards, S. et al. Standards and guidelines for the interpretation of sequence variants: a joint consensus recommendation of the American college of medical genetics and genomics and the association for molecular pathology. Genet. Med. 17, 405-424 (2015).

20. T2D-GENES Consortium, GoT2D Consortium, DIAGRAM Consortium. Available at: http://www.type2diabetesgenetics.org/gene/geneInfo/RFX6. Accessed on 25 November 2016.

21. Suzuki, K. et al. Transcriptional regulatory factor $\times 6(\mathrm{Rf} \times 6)$ increases gastric inhibitory polypeptide (GIP) expression in enteroendocrine K-cells and is involved in GIP hypersecretion in high fat diet-induced obesity. J. Biol. Chem. 288, 1929-1938 (2013)

22. MacArthur, D. G. et al. A systematic survey of loss-of-function variants in human protein-coding genes. Science 335, 823-828 (2012).

23. Huopio, H. et al. Clinical, genetic, and biochemical characteristics of early-onset diabetes in the finnish population. J. Clin. Endocrinol. Metab. 101, 3018-3026 (2016).

24. Skopkova, M. et al. Two novel RFX6 variants in siblings with Mitchell-Riley syndrome with later diabetes onset and heterotopic gastric mucosa. Eur. J. Med. Genet. 59, 429-435 (2016).

25. Manrai, A. K. et al. Genetic misdiagnoses and the potential for health disparities. N. Engl. J. Med. 375, 655-665 (2016).

26. Shields, B. M. et al. Maturity-onset diabetes of the young (MODY): how many cases are we missing? Diabetologia 53, 2504-2508 (2010).

27. Chheda, H. et al. Whole-genome view of the consequences of a population bottleneck using 2926 genome sequences from Finland and United Kingdom. Eur. J. Hum. Genet. 25, 477-484 (2017).

28. Lim, E. T. et al. Distribution and medical impact of loss-of-function variants in the Finnish founder population. PLoS Genet. 10, e1004494 (2014).

29. Akinrinade, O., Koskenvuo, J. W. \& Alastalo, T.-P. Prevalence of Titin truncating variants in general population. PLOS ONE 10, e0145284 (2015).

30. Concepcion, J. P. et al. Neonatal diabetes, gallbladder agenesis, duodenal atresia, and intestinal malrotation caused by a novel homozygous mutation in RFX6. Pediatr. Diabetes 15, 67-72 (2014)

31. Spiegel, R. et al. Clinical characterization of a newly described neonatal diabetes syndrome caused by RFX6 mutations. Am. J. Med. Genet. 155A, 2821-2825 (2011). 
32. Chappell, L. et al. A further example of a distinctive autosomal recessive syndrome comprising neonatal diabetes mellitus, intestinal atresias and gall bladder agenesis. Am. J. Med. Genet. 146A, 1713-1717 (2008).

33. Chandra, V. et al. RFX6 regulates insulin secretion by modulating $\mathrm{Ca}^{2+}$ homeostasis in human $\beta$ Cells. Cell Rep. 9, 2206-2218 (2014).

34. Sansbury, F. H. et al. Biallelic RFX6 mutations can cause childhood as well as neonatal onset diabetes mellitus. Eur. J. Hum. Genet. 23, 1744-1748 (2015).

35. Zegre Amorim, M. et al. Mitchell-riley syndrome: a novel mutation in RFX6 gene. Case Rep. Genet. 2015, 937201 (2015).

36. Mitchell, J. et al. Neonatal diabetes, with hypoplastic pancreas, intestinal atresia and gall bladder hypoplasia: search for the aetiology of a new autosomal recessive syndrome. Diabetologia 47, 2160-2167 (2004).

37. Cooper, D. N., Krawczak, M., Polychronakos, C., Tyler-Smith, C. \& Kehrer-Sawatzki, H. Where genotype is not predictive of phenotype: towards an understanding of the molecular basis of reduced penetrance in human inherited disease. Hum. Genet. 132, 1077-1130 (2013).

38. Murphy, R., Ellard, S. \& Hattersley, A. T. Clinical implications of a molecular genetic classification of monogenic beta-cell diabetes. Nat. Clin. Pract. Endocrinol. Metab. 4, 200-213 (2008).

39. Besser, R. E. J. et al. Urinary C-peptide creatinine ratio is a practical outpatient tool for identifying hepatocyte nuclear factor 1-/hepatocyte nuclear factor 4maturity-onset diabetes of the young from long-duration type 1 diabetes. Diabetes Care 34, 286-291 (2011).

40. Maestro, M. A. et al. Distinct roles of HNF1beta, HNF1alpha, and HNF4alpha in regulating pancreas development, beta-cell function and growth. Endocr. Dev. 12, 33-45 (2016).

41. Piccand, J. et al. Rfx6 maintains the functional identity of adult pancreatic $\beta$ cells. CellRep. 9, 2219-2232 (2014).

42. Nauck, M. A., Bartels, E., Orskov, C., Ebert, R. \& Creutzfeldt, W. Additive insulinotropic effects of exogenous synthetic human gastric inhibitory polypeptide and glucagon-like peptide-1-(7-36) amide infused at nearphysiological insulinotropic hormone and glucose concentrations. J. Clin. Endocrinol. Metab. 76, 912-917 (1993).

43. Calanna, S. et al. Secretion of glucagon-like peptide- 1 in patients with type 2 diabetes mellitus: systematic review and meta-analyses of clinical studies. Diabetologia 56, 965-972 (2013).

44. Calanna, S. et al. Secretion of glucose-dependent insulinotropic polypeptide in patients with type 2 diabetes: systematic review and meta-analysis of clinical studies. Diabetes Care 36, 3346-3352 (2013).

45. Vilsbøll, T. et al. Incretin secretion in relation to meal size and body weight in healthy subjects and people with type 1 and type 2 diabetes mellitus. J. Clin. Endocrinol. Metab. 88, 2706-2713 (2003).

46. Østoft, S. H. et al. Incretin effect and glucagon responses to oral and intravenous glucose in patients with maturity-onset diabetes of the young--type 2 and type 3. Diabetes 63, 2838-2844 (2014).

47. Abecasis, G. R., Cherny, S. S., Cookson, W. O. \& Cardon, L. R. Merlin--rapid analysis of dense genetic maps using sparse gene flow trees. Nat. Genet. 30, 97-101 (2002).

48. Kong, A. \& Cox, N. J. Allele-sharing models: LOD scores and accurate linkage tests. Am. J. Hum. Genet. 61, 1179-1188 (1997).

\section{Acknowledgements}

K.A.P. has a postdoctoral fellowship funded by the Wellcome Trust $(110082 / \mathrm{Z} / 15 / \mathrm{Z})$. S.E.F. has a Sir Henry Dale Fellow-ship jointly funded by the Wellcome Trust and the Royal Society $(105636 / Z / 14 / Z)$. S.E. and A.T.H. are Wellcome Trust Senior Investigators (WT098395/Z/12/Z), and A.T.H. is also supported by a NIHR Senior Investigator award.
M.N.W. is supported by the Wellcome Trust Institutional Strategic Support Fund (WT097835MF) and the Medical Research Council (MR/M005070/1). M.S. is supported by the NIHR Exeter Clinical Research Facility. Additional support came from the University of Exeter and the NIHR Exeter Clinical Research Facility. M.C. is supported by the European Union's Horizon 2020 research and innovation programme, project T2DSystems, under grant agreement No 667191, and the Fonds National de la Recherche Scientifique (FNRS) and Actions de Recherche Concertées de la Communauté Française (ARC), Belgium. The FINNMODY and PPP-Botnia Studies have been financially supported by the Sigrid Juselius Foundation, the Folkhalsan Research Foundation, Helsinki University Central Hospital Research Foundation, Finnish Diabetes Research Foundation, Finnish Medical Society, Foundation for Pediatric Research, Ahokas Foundation, Ollqvist Foundation, Nordic Center of Excellence in Disease Genetics, EU-EXGENESIS), Signe and Ane Gyllenberg Foundation, Swedish Cultural Foundation in Finland, Finnish Diabetes Research Foundation, Foundation for Life and Health in Finland, Paavo Nurmi Foundation, Perklén Foundation, Närpes Health Care Foundation, and Diabetes Wellness Foundation. The study has also been supported by the Municipal Heath Care Center and Hospital in Jakobstad, Health Care Centers in Vasa, Närpes and Korsholm. The views expressed are those of the authors and not necessarily those of the Wellcome Trust, the National Health Service, the NIHR, or the Department of Health.

\section{Author contributions}

K.A.P., J.K., M.C. and M.N.W. researched data and performed statistical analyses. K.A.P., J.K., M.C. and T.T. wrote the first draft of the manuscript, which was modified by all authors. All authors contributed to the discussion and reviewed or edited the manuscript. M.N.W., M.C. and T.T. are the guarantors of this work and, as such, had full access to all the data in the study and take responsibility for the integrity of the data and the accuracy of the data analysis.

\section{Additional information}

Supplementary Information accompanies this paper at doi:10.1038/s41467-017-00895-9.

Competing interests: The authors declare no competing financial interests.

Reprints and permission information is available online at http://npg.nature.com/ reprintsandpermissions/

Publisher's note: Springer Nature remains neutral with regard to jurisdictional claims in published maps and institutional affiliations.

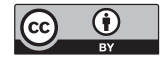

Open Access This article is licensed under a Creative Commons Attribution 4.0 International License, which permits use, sharing, adaptation, distribution and reproduction in any medium or format, as long as you give appropriate credit to the original author(s) and the source, provide a link to the Creative Commons license, and indicate if changes were made. The images or other third party material in this article are included in the article's Creative Commons license, unless indicated otherwise in a credit line to the material. If material is not included in the article's Creative Commons license and your intended use is not permitted by statutory regulation or exceeds the permitted use, you will need to obtain permission directly from the copyright holder. To view a copy of this license, visit http://creativecommons.org/ licenses/by/4.0/.

(C) The Author(s) 2017 